\title{
Murine Insulinoma Cell-Conditioned Medium with B ETA2/Neurod1 Transduction Efficiently Induces the Differentiation of Adipose-Derived Mesenchymal Stem Cells into $\beta$-Like Cells both In Vitro and In Vivo
}

Koichi Kawamoto"\#, Shigeharu Yabe ${ }^{2 \#, ~ M a s a m i t s u ~ K o n n o ~}{ }^{3, \#}$, Hideshi Ishii ${ }^{3}$, Naohiro Nishida ${ }^{3}$, Jun Koseki ${ }^{3}$, Satsuki Fukuda ${ }^{2}$, Yoshito Tomimaru ${ }^{1}$, Naoki Hama ${ }^{1}$, Hiroshi Wada ${ }^{1}$, Shogo Kobayashi ${ }^{1}$, Hidetoshi Eguchi ${ }^{1}$, Masahiro Tanemura ${ }^{4}$, Toshinori Ito ${ }^{5}$, Eun Young Lee ${ }^{6}$, Eri Mukai $^{6}$, Takashi Miki ${ }^{6}$, Yuichiro Doki ${ }^{1}$, Masaki Mori ${ }^{1}$, Tatsuo S Hamazaki², Hiroaki Nagano ${ }^{1 *}$ and Hitoshi Okochi $^{2 *}$

${ }^{1}$ Department of Surgery, Graduate School of Medicine, Osaka University, 2-2 Yamadaoka, Suita, Osaka, 565-0871, Japan

${ }^{2}$ Department of Regenerative Medicine, Research Institute, National Center for Global Health and Medicine, 1-21-1 Toyama, Shinjuku-ku, Tokyo 162-8655, Japan ${ }^{3}$ Department of Frontier Science for Cancer and Chemotherapy, National Hospital Organization Kure Medical Center, Hiroshima, 737-0023, Japan

${ }^{4}$ Department of Surgery and Institute for Clinical Research, National Hospital Organization Kure Medical Center, Hiroshima, 737-0023, Japan

${ }^{5}$ Department of Complementary and Alternative Medicine, Graduate School of Medicine, Osaka University, 2-2 Yamadaoka, Suita, Osaka, 565-0871, Japan

${ }^{6}$ Department of Medical Physiology, Chiba University, Graduate School of Medicine, 1-8-1 Inohana, Chuo-ku, Chiba, 260-8670, Japan

"These authors contributed equally to the work

\begin{abstract}
Background: Mesenchymal stem cells (MSCs), including adipose tissue-derived mesenchymal stem cells (ADSCs), are multipotent and can differentiate into various cell types, including pancreatic $\beta$ cells. Therefore, ADSCs present a potential cell source for the treatment of type 1 diabetes mellitus (T1DM). However, current in vitro protocols are insufficient to induce fully matured insulin-producing $\beta$ cells. In this study, we assessed the effectiveness of overexpression of BETA2 (NeuroD1), a member of the basic helix-loop-helix transcription factor family, with murine insulinoma cell line-derived conditioned medium (MIN6-CM) to improve the differentiation capacity of ADSCs into insulin-producing cells.
\end{abstract}

Method: Murine ADSCs were isolated from C57BL/6 mice, transduced with several transcriptional factors (TFs), and stable transfectants were established. MIN6-CM was prepared. Syngeneic recipient mice were rendered diabetic by a single injection of streptozotocin, and differentiated cells were transplanted under the kidney capsule of recipient mice. Next, blood glucose levels were monitored.

Results: CM alone was sufficient to induce insulin mRNA expression in vitro. However, other TFs were not detected. ADSCs cultured with MIN6-CM induced insulin expressions in vitro, but other $\beta$ cell-related TFs were been detected. However, BETA2 transduction in MIN6-CM resulted in robust expression of multiple $\beta$ cell phenotypic markers. Moreover, insulin content analysis revealed insulin protein expression in vitro. Furthermore, in vivo transplant studies revealed the effectiveness of the simultaneous use of BETA2 transduction with the CM.

Conclusion: These results suggest that the balance of cytokines and growth factors in addition to gene manipulation would benefit the efficient differentiation of ADSCs into pancreatic $\beta$ cells. Our technology could provide a path to $\beta$ cell differentiation and novel cell replacement-based therapies for T1DM.

Keywords: Adipose tissue-derived stem cell (ADSC); BETA2 (NeuroD1); Conditioned medium; Transcription factors; $\beta$ cells

Abbreviations: ADSC: Adipose Tissue-derived Mesenchymal Stem Cell; CM: Conditioned Medium; iPS: Induced Pluripotent Stem

\section{Introduction}

Type 1 diabetes mellitus (T1DM) typically manifests in childhood and has been estimated to account for $5-10 \%$ of all diagnosed cases of diabetes. T1DM is characterized by absolute insulin deficiency due to immunologicaldestruction of insulin-secreting pancreatic $\beta$ cells [1]. The limitation of insulin therapy has been well documented [2]. Therefore, $\beta$ cell replacement therapy is required for treatment of such patients [3]. Pancreas or islet transplantations are viable treatment options for T1DM $[4,5]$. However, the side-effects of immunosuppressants cannot be disregarded. Moreover, transplantation on a large scale is limited by the availability of pancreas donors. Thus, $\beta$ cell regeneration from auto tissue has been proposed as the best strategy to treat diabetes. Almost all $\beta$ cells are destroyed in patients with established T1DM, stimulating the researcher to produce $\beta$ cells from other cell types.

Cellular plasticity has been extensively investigated for possible roles in the propagation of pancreatic $\beta$ cells. Earlier studies have shown that ectopic expression of pancreatic and duodenal homeobox-1 ( $p d x 1)$ is sufficient to induce expression of insulin in murine liver cells [6]. More recently, since the introduction of induced pluripotent stem cells (iPS), researchers are making a concerted effort again to demonstrate

${ }^{*}$ Corresponding authors: Hitoshi Okochi, MD, PhD, Professor, Department of Regenerative Medicine, Research Institute, National Center for Global Health and Medicine, 1-21-1 Toyama, Shinjuku-ku, Tokyo 162-8655, Japan, Tel: +81-(0)33202-7181-2727; Fax: +81- 03-3207-1038; E-mail: hokochi@ri.ncgm.go.jp

Hiroaki Nagano, MD, PhD, Associate Professor, Department of Surgery, Osaka University, Graduate School of Medicine, Suita, Yamadaoka 2-2, Osaka 5650871, Japan, Tel: +81-(0)6-6879-3251; Fax: +81-(0)6-6879-3259; E-mail: hnagano@gesurg.med.osaka-u.ac.jp

Received July 10, 2014; Accepted July 26, 2014; Published July 28, 2014

Citation: Kawamoto K, Yabe S, Konno M, Ishii H, Nishida N, et al. (2014) Murine Insulinoma Cell-Conditioned Medium with BETA2/Neurod1 Transduction Efficiently Induces the Differentiation of Adipose-Derived Mesenchymal Stem Cells into $\beta$-Like Cells both In Vitro and In Vivo. J Stem Cell Res Ther 4: 221. doi:10.4172/21577633.1000221

Copyright: (c) 2014 Kawamoto K, et al. This is an open-access article distributed under the terms of the Creative Commons Attribution License, which permits unrestricted use, distribution, and reproduction in any medium, provided the original author and source are credited. 
Citation: Kawamoto K, Yabe S, Konno M, Ishii H, Nishida N, et al. (2014) Murine Insulinoma Cell-Conditioned Medium with BETA2/Neurod1 Transduction Efficiently Induces the Differentiation of Adipose-Derived Mesenchymal Stem Cells into $\beta$-Like Cells both In Vitro and In Vivo. J Stem Cell Res Ther 4: 221. doi:10.4172/2157-7633.1000221

Page 2 of 9

the plasticity of terminally differentiated cells [7]. In the case of pancreatic cell reprogramming, Melton et al demonstrated that acinar cells could be reprogrammed into $\beta$ cells using a specific combination of three transcription factors (TFs), namely Pdx1, neurogenin 3 (Ngn3), and v-maf musculoaponeurotic fibrosarcoma oncogene homolog $\mathrm{A}$ (MafA) [8]. Furthermore, glucagon-producing pancreatic a cells have also been shown to be reprogrammable into $\beta$ cells $[9,10]$. These results demonstrated that various cell types present potential cell sources for the treatment of T1DM. However, in vivo reprogramming strategies have several limitations, such as the risk of viral transmission and low reprogramming efficiency for clinical use.

Stem cells, such as embryonic stem cells, as iPS cells, present another attractive source for tissue engineering because they have the capability of self-renewal. Originally, embryonic stem cells held promise as a source of renewable $\beta$ cells, but their potential has proven more difficult than expected. Alternatively, pancreatic progenitor cells present as possible candidates [11]. Multipotent mesenchymal stem cells (MSCs), which represent a nonhematopoietic cell population, can also differentiate into mesenchymal tissues (i.e., bone, cartilage, or fat) [12]. MSCs were first isolated from bone marrow and later from nonmarrow tissues, including umbilical cord blood and adipose tissue, and are clinically applicable because of easy accessibility and large scale preparation. Thus, adipose tissue-derived mesenchymal stem cells (ADSCs) have been also explored as a possible source of pancreatic $\beta$ cells.

During vertebrate embryogenesis, the development of pancreas is regulated by the sequential expression of a molecular network of TFs [13]. It is generally accepted that several defined factors are needed in the differentiation of insulin-producing cells [13]. Among them, Pdx1 is thought to be a key transcriptional regulator of endocrine, acinar, and ductal cell development. PDX-1-deficient mice die rapidly after birth due to pancreatic insufficiency [14]. Previously, our research group demonstrated that Pdx1-transfected ADSCs can mature in vivo [15]. The combined expression of Pdx1 and MafA with either Ngn3 or NeuroD are required for both exocrine cell reprogramming and staging differentiation [16]. However, simple transduction of several TFs could not induce $\beta$ cell maturation. NeuroD1 is a member of the NeuroD family and binds to the E element of the insulin gene [17] to modulate the expression of genes, such as SUR1, which forms $\mathrm{K}^{+}$channels with Kir6.2 to regulate insulin secretion [18]. The BETA2/NeuroD protein, a class B bHLH TF, has been cloned as a transcriptional activator of the insulin gene [4] and neurogenic factor in Xenopus embryos [2].

Several studies have already found that MSCs have an immunomodulatory effect by both contact-dependent and -independent mechanisms [19-24], and they secrete or release many substances into the surrounding medium. This phenomenon stimulated researchers to use conditioned medium (CM) of MSCs to reduce the toxic effects of ischemia reperfusion injury [25]. We hypothesized that the cocktail of cytokine and growth factors produced by an insulinoma cell line could efficiently induce $\beta$ cell differentiation into insulin-producing cells because such cells retain the expression of multiple insulin-related genes, including pdx1, paired box protein (pax6), kir6.2, and ins2.

To determine the impact of MIN6-CM on the induction of ADSC differentiation into insulin-producing cells, we cultured ADSCs in MIN6-CM. Here we report that CM per se was sufficient to induce insulin detection by quantitative real-time reverse transcriptase polymerase chain reaction (qRT-PCR). Next, we assessed whether the combinatorial effect of stable BETA2 transfection with $\mathrm{CM}$ would be a useful method for the induction of insulin-producing $\beta$ cells. Stable expression of BETA2 in ADSCs in MIN6-CM induces a pancreatic phenotype in vitro. The transplantation of these cells into streptozotocin (STZ)-induced diabetic mice results in engraftment of the transplanted BETA2-ADSCs in the pancreas, leading to the amelioration of hyperglycemia.

\section{Materials and Methods}

\section{ADSC isolation}

Murine ADSCs were isolated from C57BL/6 mice as previously described [26]. In brief, adipose tissue was washed twice with Dulbecco's phosphate-buffered saline (D-PBS; Invitrogen, Carlsbad, CA, USA) containing $50 \mathrm{U} / \mathrm{mL}$ of penicillin and $50 \mu \mathrm{g} / \mathrm{mL}$ of streptomycin (Invitrogen) and were then cut into fine pieces, which were incubated with D-PBS containing $1.0 \mathrm{mg} / \mathrm{mL}$ of Clostridium histolyticum collagenase (Sigma-Aldrich, St. Louis, MO, USA) in a $37^{\circ} \mathrm{C}$ shaking incubator for $1 \mathrm{~h}$. The tissue was filtered through a sterile $70-\mu \mathrm{m}$ nylon mesh and resuspended in Dulbecco's modified Eagle's medium (DMEM; (Nacalai Tesque, Kyoto, Japan) supplemented with 10\% fetal bovine serum (FBS; HyClone; Thermo Scientific, Waltham, MA, USA), penicillin, and streptomycin.

\section{Preparation of CM}

To generate CM, a murine insulinoma cell line, MIN6 cells were allowed to grow to $50 \%$ confluence with standard DMEM with $10 \%$ FBS, PSM, sodium pyruvate, and 2-mercaptoethanol (Nacalai Tesque, Inc., Kyoto, Japan) in 150-mm dishes (Figure 1A). Next, DMEM was replaced with a new media on day 2 . After a 48 -h culture period, the medium was collected (MIN6-CM\#1) and replaced to a fresh medium. Then, CMs were collected every 48-h incubation. CM\#1, CM\#2, and $\mathrm{CM} \# 3$ were pooled and filtered using a bottle-top filter (Corning Incorporated, Corning, NY, USA) to remove cells and debris. CM samples were frozen at $-20^{\circ} \mathrm{C}$ for later use. MIAPaCa-2 and PANC1 cell lines were grown in complete medium supplemented with $10 \%$ FBS to produce pancreatic cancer cell line-derived CM. MIAPaCa-2derived CM (MIAPaCa-CM) and PANC-1-derived CM (PANC-CM) were also prepared using the same protocol.

\section{Quantitative real-time reverse transcription polymerase chain reaction}

For mRNA detection, total RNA from both pre- and post-treated cells were extracted in TRIzol Reagent (Invitrogen), and cDNA was amplified by qRT-PCR. $\beta$-actin was used as an internal control for normalization. All reactions were performed along with negative (water) and positive (pancreatic cells) controls.

\section{Immunostaining}

For immunocytochemical analysis, cells were plated in a $60-\mathrm{mm}$ dish, washed twice with D-PBS, and fixed with $4 \%$ paraformaldehyde for $20 \mathrm{~min}$ at room temperature. Then, the dishes were washed three times with D-PBS and treated with $0.5 \%$ Triton X-100 in PBS for 15 min. The cells were treated with 3\% BSA-PBS for $60 \mathrm{~min}$ and stained with C-peptide (1:400 dilution; Cell Signaling Technology \#4593, Danvers, MA, USA) for $16 \mathrm{~h}$ at $4^{\circ} \mathrm{C}$, and then treated with a second antibody (AlexaFluor 647) for $60 \mathrm{~min}$ at room temperature. Nuclei were counter-stained with 4',6-diamidino-2-phenylindole (DAPI). 
Citation: Kawamoto K, Yabe S, Konno M, Ishii H, Nishida N, et al. (2014) Murine Insulinoma Cell-Conditioned Medium with BETA2/Neurod1 Transduction Efficiently Induces the Differentiation of Adipose-Derived Mesenchymal Stem Cells into $\beta$-Like Cells both In Vitro and In Vivo. J Stem Cell Res Ther 4: 221. doi:10.4172/2157-7633.1000221

A

$\begin{array}{ccccc}\begin{array}{c}\text { Passage } \\ \text { (Day 0) }\end{array} & \begin{array}{c}\text { Media changed } \\ \text { (Day 2) }\end{array} & \begin{array}{c}\text { Collection of } \\ \text { CM \#1 (Day 4) }\end{array} & \begin{array}{c}\text { Collection of } \\ \text { CM \#2 (Day 6) }\end{array} & \begin{array}{c}\text { Collection of CM \#3 } \\ \text { Passage (Day 8) }\end{array}\end{array}$

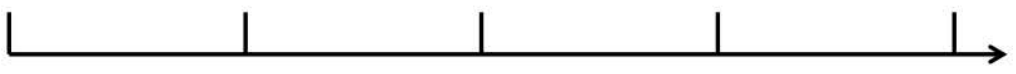

B

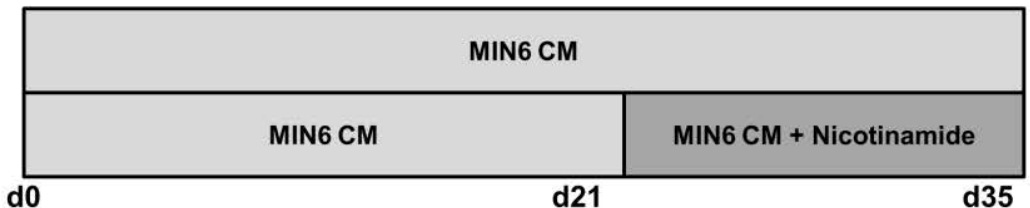

C

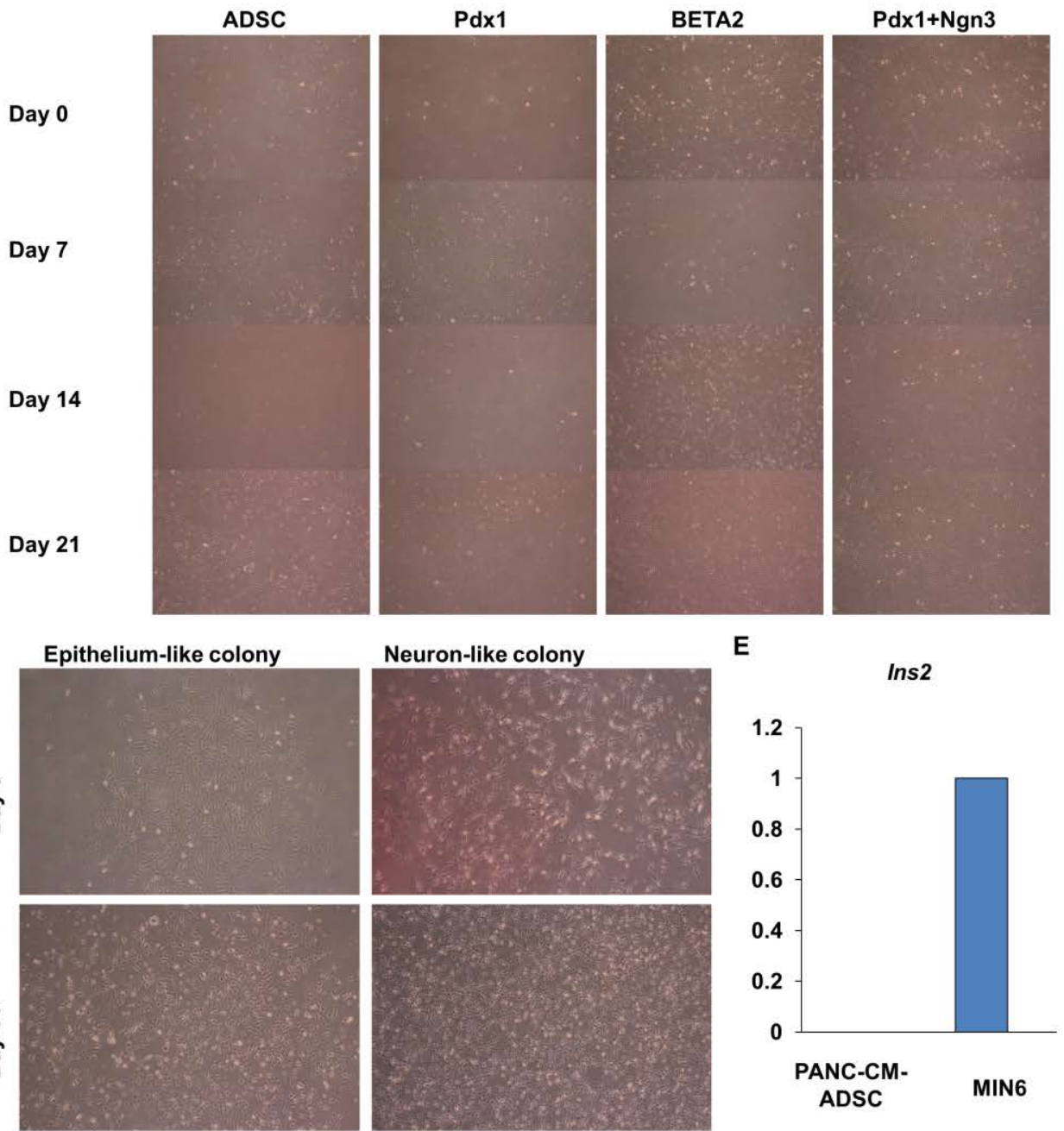

Figure 1: Schematic representation of this study. (A) Preparation of conditioned medium (CM). (B) adipose-derived mesenchymal stem cells (ADSCs) or genemanipulated ADSCs were cultured in murine insulinoma cell line-derived CM (MIN6-CM) for 3 weeks and then divided into two groups. (C) Morphological change of ADSCs and subclones cultured with MIN6 conditioned medium. (D) ADSC with PANC-CM. (E) Polymerase chain reaction (PCR) analysis of Ins2.

Insulin content measurements and glucose-stimulated insulin release (GSIS)

For insulin content and GSIS analysis, cells were seeded in a 6-well plate, cultured for $16 \mathrm{~h}$, and then placed for $30 \mathrm{~min}$ in medium containing $3 \mathrm{mM}$ glucose (Lo glucose), which was then replaced with a media containing the various constituents indicated and incubated again for an additional $60 \mathrm{~min}$. The supernatant was frozen for later use. The cells were then lysed with acid ethanol at $4^{\circ} \mathrm{C}$ for $16 \mathrm{~h}$. Cell lysates were collected and stored at $-80^{\circ} \mathrm{C}$. Insulin levels were then determined in both GSIS and intracellular contents using the ultra-sensitive "PLUS" mouse insulin ELISA kit (Morinaga Institute of Biological Science, Inc., Yokohama, Japan). TMB (3,3',5,5'-tetramethylbenzidine) solution was added to each well, and the plates were then incubated for 
Citation: Kawamoto K, Yabe S, Konno M, Ishii H, Nishida N, et al. (2014) Murine Insulinoma Cell-Conditioned Medium with BETA2/Neurod1 Transduction Efficiently Induces the Differentiation of Adipose-Derived Mesenchymal Stem Cells into $\beta$-Like Cells both In Vitro and In Vivo. J Stem Cell Res Ther 4: 221. doi:10.4172/2157-7633.1000221

Page 4 of 9

40 min, until the reaction was terminated by the addition of an equal volume of stopping solution; optical density was determined at 450/630 nm (PerkinElmer, Waltham, MA, USA).

\section{FACS analysis}

Cells were treated with Accutase reagent (EMD Millipore, Billerica, MA, USA) to dissociate the cells, which were then resuspended in staining media [DPBS supplemented with FBS (1\%) and EDTA (2 $\mathrm{mM})$ ]. Next, the cells were stained with fluorescein isothiocyanateconjugated anti-mouse CD71 (Clone C2; BD Pharmingen, Franklin Lakes, NJ, USA), phycoerythrin-conjugated anti-mouse CD31 (eBioscience, San Diego, CA, USA), APC-anti mouse CD140a (Clone APA5; BioLegend, San Diego, CA, USA), eFluor450 anti-mouse CD45, and allophycocyanin-conjugated anti-mouse CD90.2 for $30 \mathrm{~min}$ on ice.

\section{Retroviral preparation}

Pdx1 cDNA was obtained as previously described [15]. The open reading frame of the human $P d x 1$ gene was integrated into the retrovirus vector pMSCV-puro. To prepare the retroviruses, PT67 cells were cultured in DMEM supplemented with 10\% FBS and PSM with puromycin. Fugene 6 transfection reagent (Promega, Madison, WI, USA) was diluted with $500 \mu \mathrm{L}$ of DMEM and incubated for $5 \mathrm{~min}$ at room temperature. Plasmid DNA $(2.5 \mu \mathrm{g})$ was added to the mixture, which was incubated for an additional $15 \mathrm{~min}$ at room temperature. Then, the culture media was replaced with fresh DMEM supplemented $10 \%$ FBS and the DNA/Fugene 6 mixture was added dropwise onto the HEK-293Ta cells. The medium was replaced after $24 \mathrm{~h}$. After an additional $48 \mathrm{~h}$, virus-containing supernatants, derived from the HEK293 Ta cultures, were filtered through a $0.22-\mu \mathrm{L}$ cellulose-acetate filter and used.

\section{Sphere formation}

To assess the impact of TFs and CMs for the induction or maintenance of $\beta$-like cells, the sphere formation protocol was employed. In brief, control or TF-transduced ADSCs were placed in an ultra-low attachment 96-well round bottom plate with hydrogel (Costar 7007) at a density of 1000 cells/well. Colonies were picked up on day 2 and plated on either gelatin-coated or uncoated Petri dishes (Sansyo Co., Ltd., Tokyo, Japan).

\section{Transplantation to diabetic mice}

Eight-12-week-old female B6 mice were housed in an airconditioned environment under a 12-h light-dark cycle. Freshly dissolved STZ (Nacalai Tesque) was used to make a concentration of $10 \mathrm{mg} / \mathrm{mL}$ stock solution. Then, the recipient mice were rendered diabetic by a single intraperitoneal injection of $200 \mathrm{mg} / \mathrm{kg} \mathrm{STZ}$ prior to transplantation. Next, blood glucose levels were monitored every other day and confirmed for hyperglycemia $(400 \mathrm{mg} / \mathrm{dL})$. The cells were transplanted under the left kidney capsular space of the recipient diabetic mice using a Hamilton syringe with a 26-gauge needle. Then, the blood glucose levels were monitored every other day using a commercial blood glucose meter.

\section{Statistical analyses}

Data are presented as means \pm standard errors of the mean of independent experiments. Statistical testing was performed using the Student's $t$-test to detect differences between groups. In cases of multiple groups testing, analysis of variance was conducted followed by a posteriori $t$-test. Differences were considered statistically significant at $p<0.05\left(^{*}\right)$ and $<0.001\left(^{* *}\right)$.

\section{Results}

\section{CM with transcription factor BETA2-induced efficient differentiation of ADSCs into multiple TF-positive $\beta$-like cells}

Figures $1 \mathrm{~A}$ and $1 \mathrm{~B}$ describe the experimental protocol used in this study. MIN6-CM was prepared as described in the Materials and Methods section. First, we evaluated the morphological changes of ADSCs and their subclones cultured with MIN6-CM. As shown in Figure 1C, the morphological differences were minimal. However, there was a trend of round morphology among the cultured BETA2ADSCs. When ADSCs were cultured with CM from the human pancreatic carcinoma cell line MIAPaCa-CM, ADSCs displayed massive apoptosis within 9 days (Data not shown). Next, we assessed the impact of PANC-CM. In contrast to MIAPaCa-CM, ADSCs can grow with PANC-CM with both epithelial-like and neuron-like colonies (Figure 1D). However, ins2 message was not detected in PANC-CM-ADSC compared to MIN6 (Figure 1E). Next, we assessed the effect of MIN6-CM on ADSC. Surprisingly, CM culture for 1 week was sufficient for insulin detection by qRT-PCR (Figure 2A). These results suggest that MIN6-CM would be useful for induction of $\beta$ cell differentiation. However, other TFs, such as pax6 and kir6.2, were not detected in this group during the overall 5-week culture period. Next, we assessed the differentiation efficiencies of MIN6-CM in combination with several TFs. As presented in Figure 2A, Pdx1-ADSC with CM was not effective to induce multiple TFs. However, BETA2-ADSCs with MIN6-CM resulted in efficient differentiation into insulin-producing cells. As early as culture day 3, both pax6 and kir6.2 were detected, and their expressions were retained. These results clearly demonstrate that BETA2 would be the best partner for CM-mediated differentiation. We further confirmed the effectiveness of this combination by qRT-PCR. $P d x$ - 1 overexpression with CM resulted in a synergistic upregulation of insulin messaging (Figure 2B). Furthermore, BETA2-ADSC with CM resulted in more effective induced insulin expression.

We next used nicotinamide for further maturation of $\beta$-like cells. As shown in Figure 2A, nicotinamide did not further enhance mRNA levels of Pax6, Kir6.2, or Ins2. Thus, nicotinamide addition was not very effective for further maturation of $\beta$-like cells. Then, we selected nicotinamide-free culture media for further experiments. For Pdx1-ADSC without MIN6-CM, insulin expression was not detected (control group), which was in accordance with the results of our previous report [12]. Next, we evaluated the MEF-feeder effect. As shown in Figure 2C, the MEF feeder impaired differentiation of $\beta$-like cells. These results suggest that MEF seemed to not partner well with MIN6-CM. An interesting observation was that no passaged group had a better outcome than the standard passaged group as it showed more intense insulin banding (Figure 2D). Furthermore, endogenous BETA2-expression was observed in the passaged group. These results demonstrated that the usual passage protocol was better for $\beta$ cell maturation.

\section{Insulin protein expression of MIN6-CM-cultured ADSCs}

Next, we detected insulin protein expression. First, we employed C-peptide immunocytochemistry to support the notion that these $\beta$-like cells produced functional insulin [27]. Immunocytochemical analysis showed that C-peptide was undetectable (Figure 3A). Neither Pdx-ADSC nor PN-ADSC with MIN6-CM expressed detectable insulin levels by immunocytochemical analysis (Data not shown). However, there were clearly significant differences in insulin content between the controls parental ADSCs and MIN6-CM-cultured BETA2-ADSCs (Figure $3 \mathrm{~B})$ under both low and high glucose conditions $(p<0.05)$. 
Citation: Kawamoto K, Yabe S, Konno M, Ishii H, Nishida N, et al. (2014) Murine Insulinoma Cell-Conditioned Medium with BETA2/Neurod1 Transduction Efficiently Induces the Differentiation of Adipose-Derived Mesenchymal Stem Cells into $\beta$-Like Cells both In Vitro and In Vivo. J Stem Cell Res Ther 4: 221. doi:10.4172/2157-7633.1000221

A

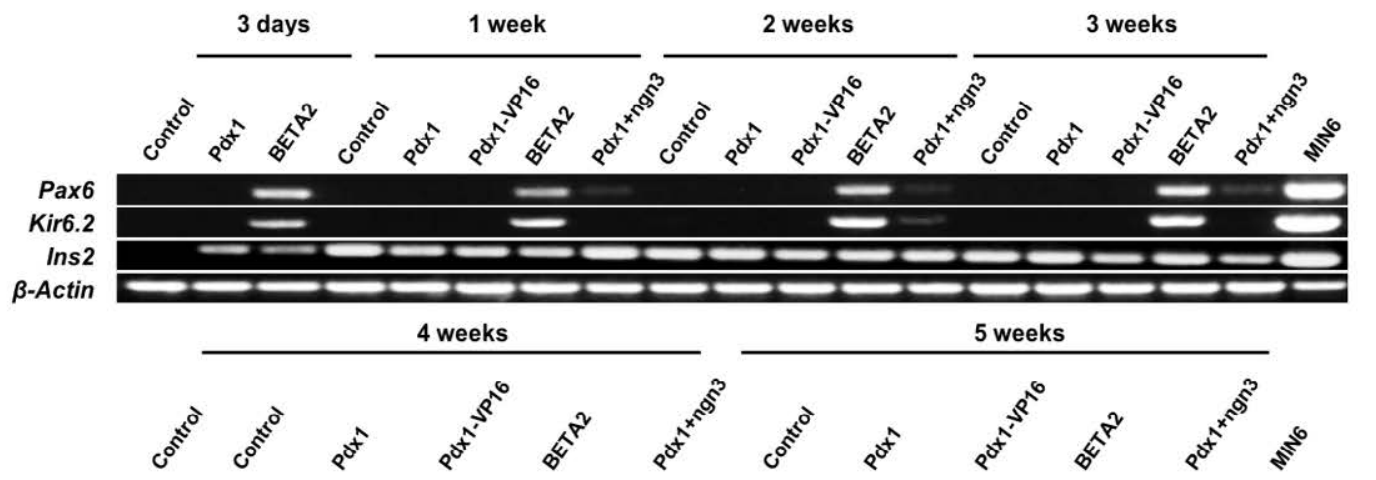

$\begin{array}{lllllllllllllllllllllll}\text { Nicotinamide } & (-) & (+) & (-) & (+) & (-) & (+) & (-) & (+) & (-) & (+) & (-) & (+) & (-) & (+) & (-) & (+) & (-) & (+) & (-) & (+)\end{array}$

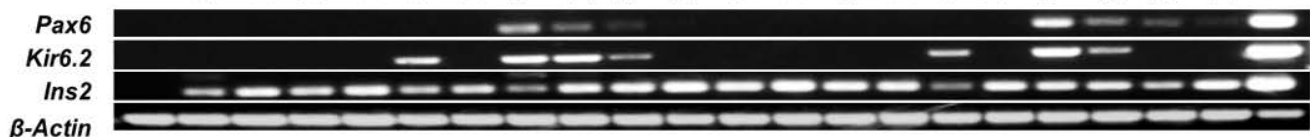

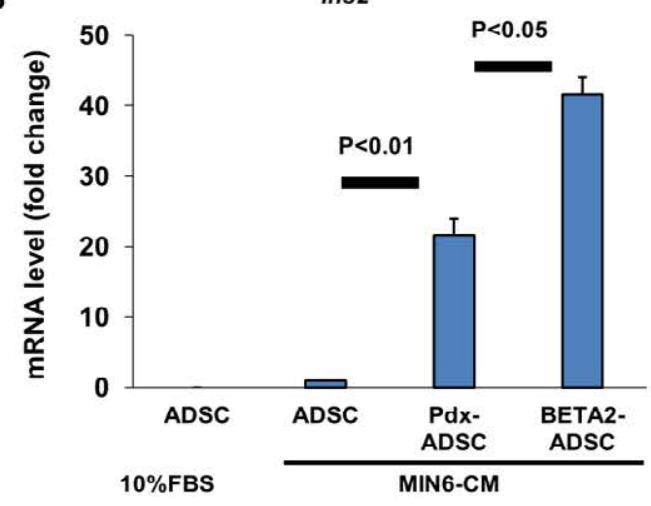

D

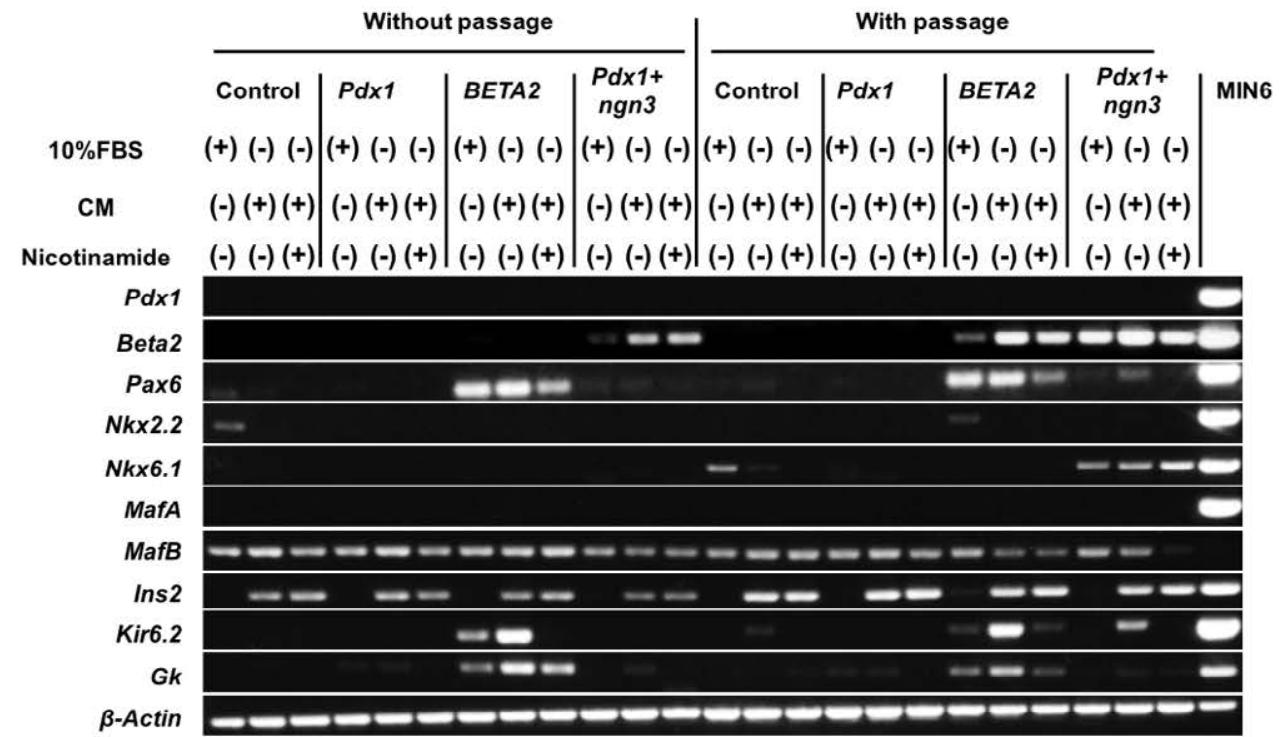

C MEF

Collagen I

$10 \%$ FBS 50\%MIN6-CM $100 \%$ MIN6-CM Pdx1+ngn3 MIN6

$(+)(-)(-)(-)(-)(-) \mid(-)(-)(-)(-)(-)$

$(-)(-)(-)(+)(+)(+)(-)(-)(+)(+)(+)$

$(+)(+)(-)(+)(-)(-)(+)(-)(+)(-)(-)$

$(-)(-)(-)(-)(+)(-)(-)(-)(-)(+)(-)$

$(-)(-)(+)(-)(-)(+)|(-)(+)(-)(-)(+)|$

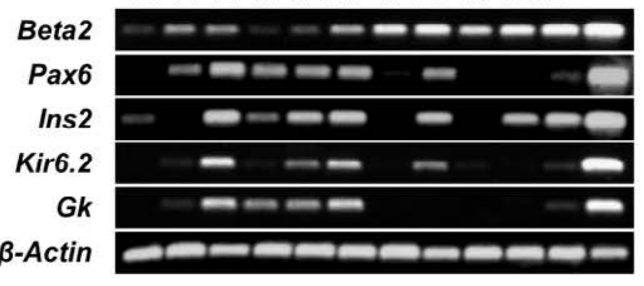

MIN6

Figure 2: (A) BETA2- adipose-derived mesenchymal stem cells (ADSCs) with murine insulinoma cell line-derived conditioned medium (MIN6-CM) induced ins2, Pax6, and Kir6.2 expression. (B) Quantitative real-time reverse transcriptase PCR analysis. (C) The effect of feeder. (D) Effect of passage to ADSC culture.

Next, we measured glucose-dependent insulin release by ADSCs in vitro. As shown in Figure 3C, MIN6-CM-cultured BETA2-ADSCs did not release insulin in response to a step-wise increase of glucose to 25 $\mathrm{mM}$ (high glucose). These results showed that insulin was detectable 
Citation: Kawamoto K, Yabe S, Konno M, Ishii H, Nishida N, et al. (2014) Murine Insulinoma Cell-Conditioned Medium with BETA2/Neurod1 Transduction Efficiently Induces the Differentiation of Adipose-Derived Mesenchymal Stem Cells into $\beta$-Like Cells both In Vitro and In Vivo. J Stem Cell Res Ther 4: 221. doi:10.4172/2157-7633.1000221
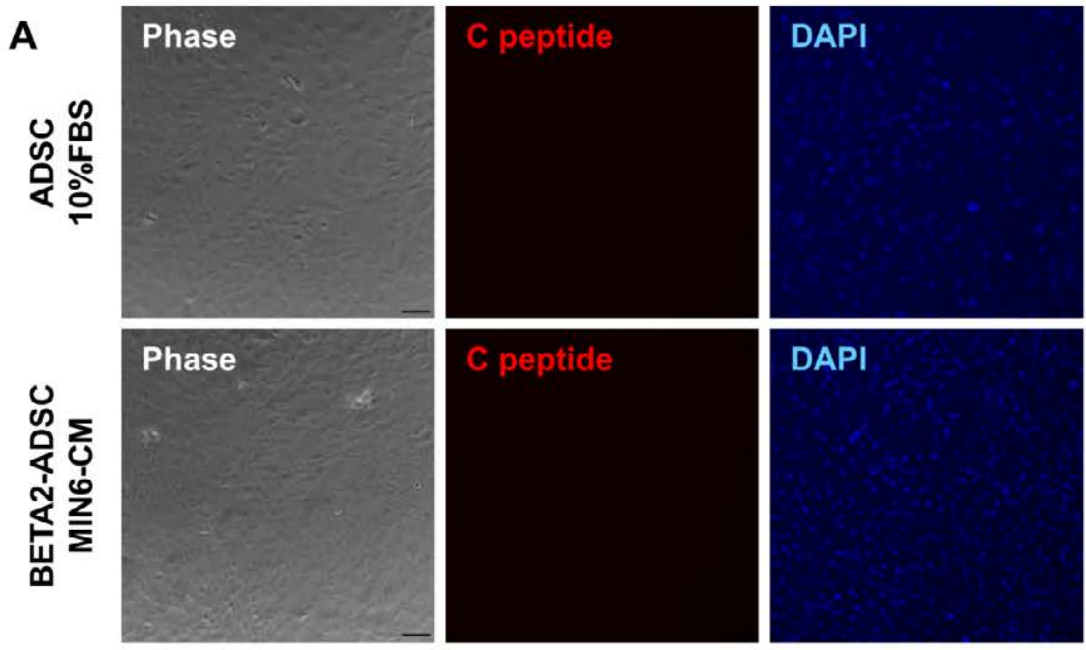

Bars $=100 \mu \mathrm{m}$

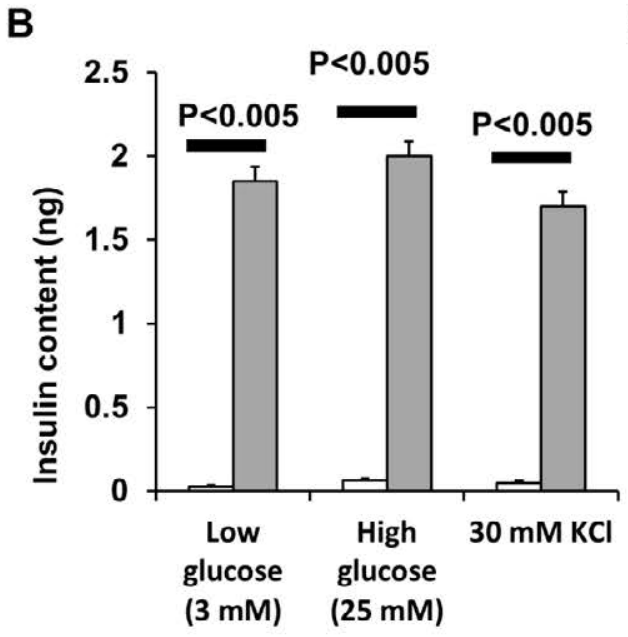

C

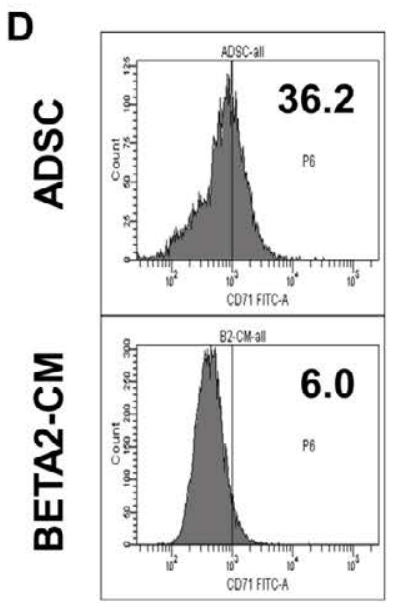

CD71

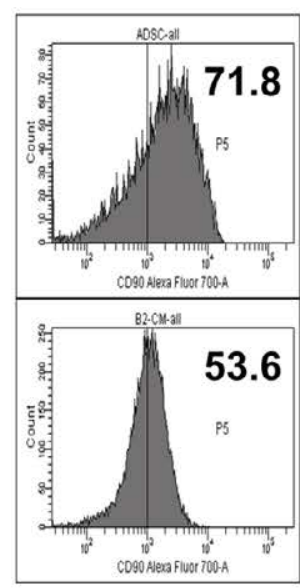

CD90

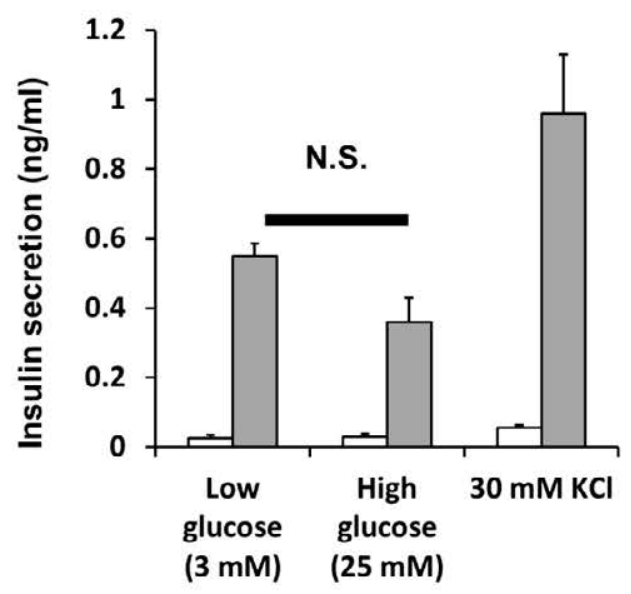

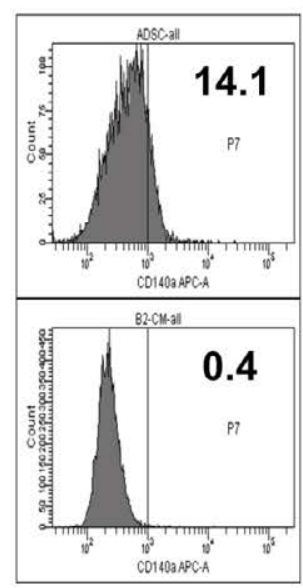

CD140a

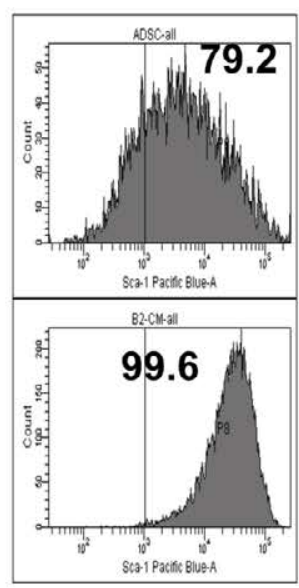

Sca-1

Figure 3: In vitro analysis of BETA2- adipose-derived mesenchymal stem cells (ADSCs) cultured with murine insulinoma cell line-derived conditioned medium (MIN6CM). (A) Immunocytochemical analysis of C-peptide. (B) Insulin content analysis. (C) Glucose-stimulated insulin secretion. (D) Surface marker analysis.

level of CD71, CD90.2, and CD140a were downregulated, whereas that of Sca-1 was upregulated. These results suggest that the MIN6-CM culture induced downregulation of mesenchymal markers. In contrast, Sca-1 seemed to be a maturation marker in our protocol.

\section{Transplantation to in vivo STZ-diabetic mice}

We investigated whether the BETA2-ADSCs with CM exhibited relevant in vivo function for the treatment of diabetes. Thus, we employed 
Citation: Kawamoto K, Yabe S, Konno M, Ishii H, Nishida N, et al. (2014) Murine Insulinoma Cell-Conditioned Medium with BETA2/Neurod1 Transduction Efficiently Induces the Differentiation of Adipose-Derived Mesenchymal Stem Cells into $\beta$-Like Cells both In Vitro and In Vivo. J Stem Cell Res Ther 4: 221. doi:10.4172/2157-7633.1000221

Page 7 of 9

a STZ-induced diabetic mouse model through the administration of 200 $\mathrm{mg} / \mathrm{kg} \mathrm{STZ}$ into the peritoneum of 8-12-week-old female B6 (Figure $4 \mathrm{~A})$. We attempted to determine whether BETA2-ADSCs with CM were capable of controlling blood glucose levels in diabetic mice. First, we assessed the diabetogenic effect of STZ. As shown in Figure 4C, STZtreated mice showed elevated blood glucose levels. Next, we evaluated whether the transplanted cells could control STZ-induced diabetes of recipient mice. As shown in Figure 4C, the recipient mice showed better blood glucose maintenance. Moreover, the beneficial effect of CM-cultured BETA2-ADSC transplantation also demonstrated a trend of better survival compared with the control group. There was no overt tumor in sacrifice mice. Thus, these transplanted ADSCs apparently did not participate in tumor formation.

\section{Sphere formation analysis}

Next, we assessed the impact of TFs and CMs to induce or maintain $\beta$-like cells. As shown in Figure 5A, ADSCs formed spheres when transferred to an ultra-low attachment 96-well round plate. However, hand-picked spheres were attached to the dish when plated in either gelatin-coated dishes (adhesive condition) or standard non-coated Petri dishes (non-adhesive condition). BETA2-ADSCs with MINCM were more adhesive compared with PDX-ADSCs with MIN-CM (Figure 5B). These results suggest the importance of BETA2 for sphere expansion. Unfortunately, C-peptide protein was not detected even with sphere formation (Figure 5C).

\section{Discussion}

Here we report a novel combination of two techniques (BETA2 transduction and MIN6-CM culture) with a great potential to refine the $\beta$ cell differentiation protocol. The balance of cytokines and growth factors in CM plays important roles in both efficient differentiation and functional maintenance of certain cell types. In this study, we found that ADSCs could express detectable insulin levels by qRT-PCR using parental MIN6-CM culture in vitro. Compared to our previous experience with $P d x 1$-ADSCs without CM, CM treatment only was enough to facilitate insulin expression in vitro. These results suggest that $\mathrm{CM}$ would be a useful induction agent for $\beta$ cell differentiation.
Furthermore, the forced expression of the BETA2 gene in murine ADSCs in the presence of MIN6-CM resulted in robust differentiation of these cells into insulin-producing cells. Using this novel technology, multiple TFs, including pax6 and kir6.2, were detected as early as culture day 3. Moreover, insulin expression of BETA2-ADSC with MIN6-CM was confirmed by insulin content analysis. Furthermore, these cells could control hyperglycemia of STZ-induced diabetic mice. These results clearly demonstrated that this combination is a promising candidate for refinement of current $\beta$ cell differentiation protocols. Of note, these effects of CM were observed only in the parenteral MIN6 cell line. When the subclones MIN6-m9 and MIN6-m14 were used, this phenomenon was not observed (data not shown). These results suggest that the selection of effective CM-producing cell offers potentially important advancements in $\beta$ cell replacement therapy.

In previous studies, we reported that Pdx1-transfected ADSCs with standard bovine serum-based medium lacked expression of pancreatic marker genes, including the two non-allelic insulin genes insulin 1 and insulin 2 [15]. These results suggested that the transduction of a single TF would be insufficient for in vitro detection of the insulin gene. However, Pdx1-ADSCs can produce insulin after in vivo maturation for 30 days when administered systemically [15]. These results suggest that in vivo maturation may contribute for further $\beta$ cell maturation, at least, in part, in our previous model. In the present study, we confirmed that Pdx1-ADSCs and BETA2-ADSCs lack ins2 expression (Figure 2). Also, the same can be said about the results of the present study from the point of the importance of in vivo maturation because BETA2ADSC with MIN6-CM resulting in insulin protein was detected only by the insulin content assay. It is noteworthy that there was a trend in the BETA2-ADSCs with CM in lower glucose levels compared with Pdx1ADSCs (historical control). In our previous study, we used intravenous tail vein cell transfer. In the present study, we employed subrenal capsular transplant, which is a more efficient site for transplantation of $\beta$-like cells. Although a proper mechanism for the effectiveness of MIN6-CM has not been clarified at present, the balance of cytokine and growth factor content may be critical for efficient $\beta$ cell differentiation. As expected, BETA2-ADSCs with MIN6-CM lost expression of the mesenchymal markers CD71, CD90, and CD140a. Recently, we

A

Transplantation

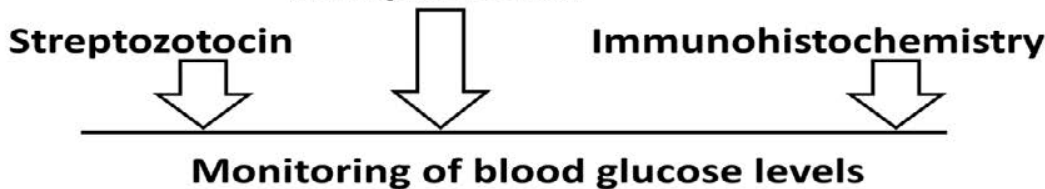

B

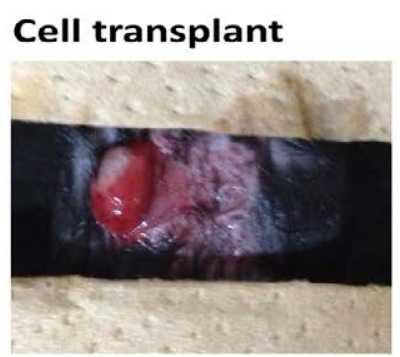

C

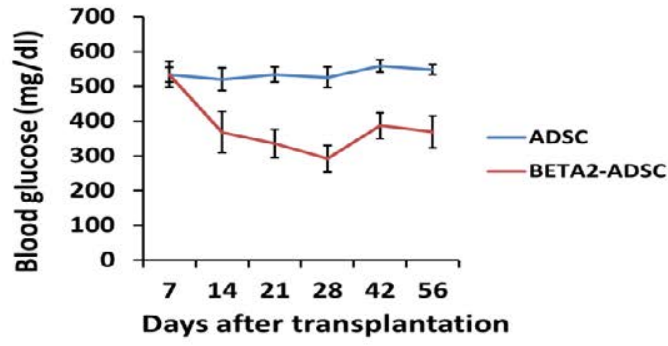

Figure 4: Blood glucose levels and survival of streptozotocin-treated diabetic mice. (A) A schematic representation of in vivo transplantation. (B) Representative cell transplant under the left kidney capsule. (C) Blood glucose levels of STZ-treated mice after cell transplantation. Comparison of blood glucose levels in ADSCtransplanted mice (blue, $n=4$ ) and BETA2-ADSC-CM-transplanted mice (red, $n=4)$. Data are presented as mean \pm SEM. 
Citation: Kawamoto K, Yabe S, Konno M, Ishii H, Nishida N, et al. (2014) Murine Insulinoma Cell-Conditioned Medium with BETA2/Neurod1 Transduction Efficiently Induces the Differentiation of Adipose-Derived Mesenchymal Stem Cells into $\beta$-Like Cells both In Vitro and In Vivo. J Stem Cell Res Ther 4: 221. doi:10.4172/2157-7633.1000221

A

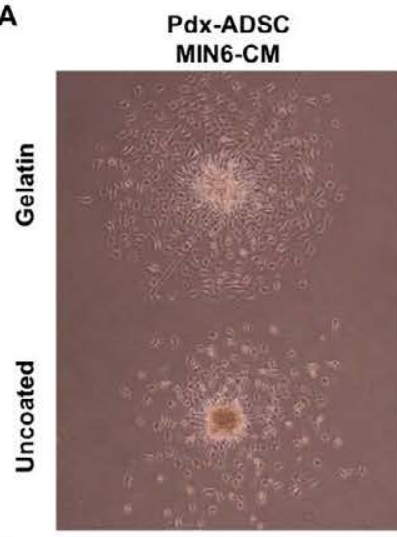

B
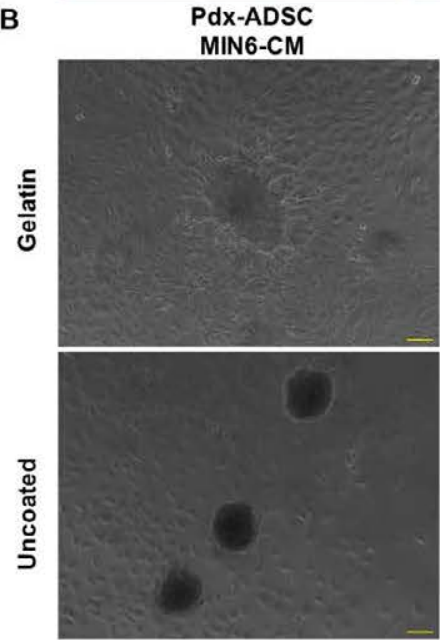

BETA2-ADSC

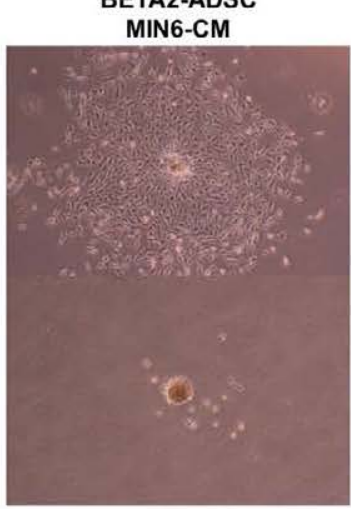

BETA2-ADSC

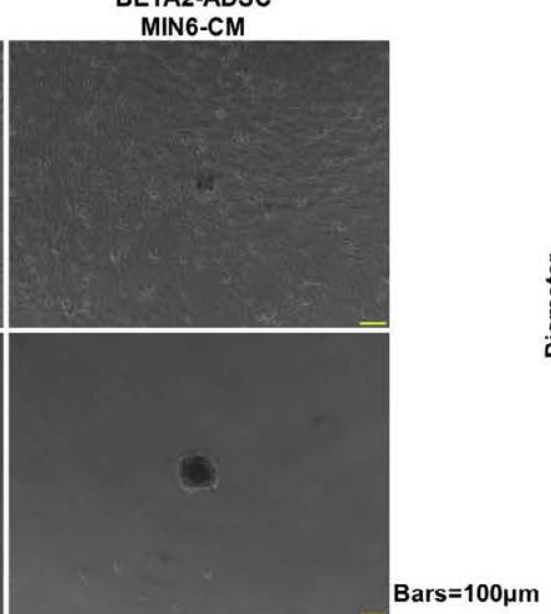

BETA2-ADSC

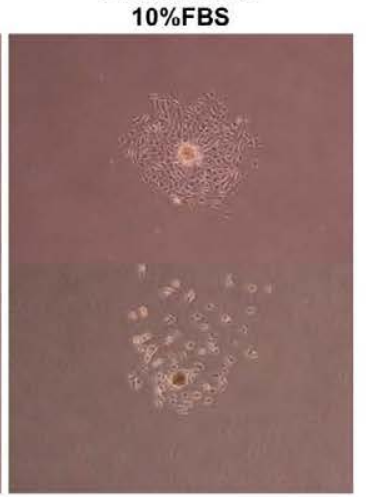

$(\mu \mathrm{m})$

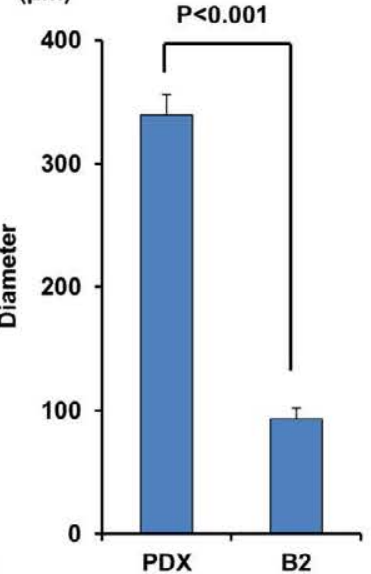

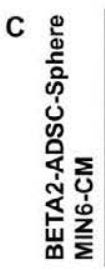
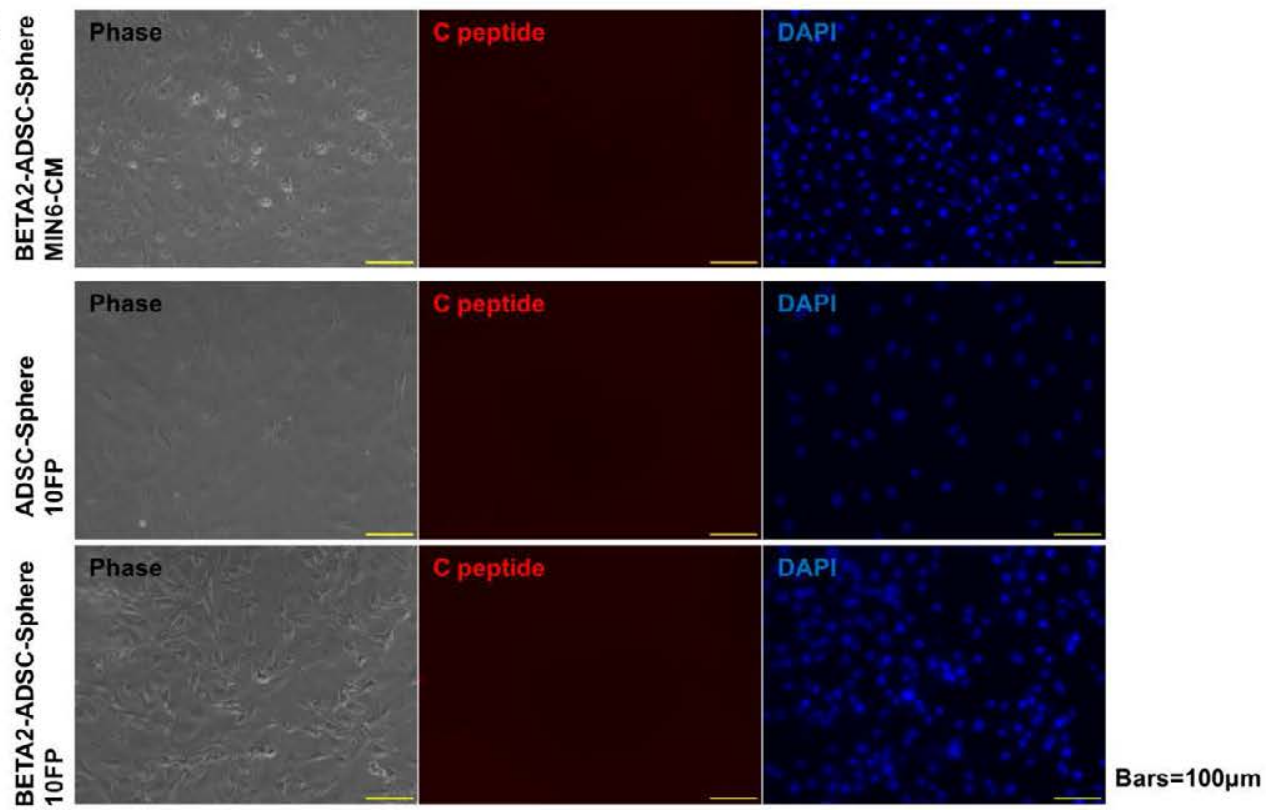

Figure 5: Sphere formation assay. (A) Sphere formation analysis on day 3. (B) Sphere formation of day 7. (C) C-peptide immunocytochemistry of sphere analysis.

reported that CD90-Hi ADSCs were reprogrammed more efficiently compared with CD90-Lo ADSCs [28]. The importance of Sca-1 has already been suggested in ductal progenitor theory [29]. In this study, Sca-1 expression was upregulated, perhaps because Sca-1 is a marker of stem and pancreatic progenitor cells. Therefore, the upregulation of Sca-1 may reflect the upregulation of progenitor properties.

A possible limitation of this study is that normoglycemia was 
Citation: Kawamoto K, Yabe S, Konno M, Ishii H, Nishida N, et al. (2014) Murine Insulinoma Cell-Conditioned Medium with BETA2/Neurod1 Transduction Efficiently Induces the Differentiation of Adipose-Derived Mesenchymal Stem Cells into $\beta$-Like Cells both In Vitro and In Vivo. J Stem Cell Res Ther 4: 221. doi:10.4172/2157-7633.1000221

not achieved in this model. The importance of MafA for pancreatic development is well recognized. Therefore, our next project is to add the factor of MafA overexpression to this combination. Another limitation of this study was the use of viral vector gene transfer. However, this and previous studies have already demonstrated that the viral-free method is clinically relevant to stem cell therapy [30-32]. A previous report also suggested that BETA2 can be transduced by non-viral methods using the protein transduction domain [33]. Thus, BETA2 overexpression using a non-viral method would present a powerful option for future clinical use. Moreover, chemicals that induce latestage $\beta$-cell differentiation have been reported [34]. Therefore, these technologies, along with our method, will further advance functional $\beta$-cell induction technology.

In summary, we successfully treated STZ-induced diabetic mice by ADSC transplantation, suggesting that this technology may open new avenues toward clinical applications of ADSCs for T1DM treatment.

\section{References}

1. Atkinson MA, Maclaren NK (1994) The pathogenesis of insulin-dependent diabetes mellitus. N Engl J Med 331: 1428-1436. [PubMed]

2. Bluestone JA, Herold K, Eisenbarth G (2010) Genetics, pathogenesis and clinical interventions in type 1 diabetes. Nature 464: 1293-1300. [PubMed]

3. Limbert C, Päth G, Jakob F, Seufert J (2008) Beta-cell replacement and regeneration: Strategies of cell-based therapy for type 1 diabetes mellitus. Diabetes Res Clin Pract 79: 389-399. [PubMed]

4. White SA, Shaw JA, Sutherland DE (2009) Pancreas transplantation. Lancet 373: 1808-1817. [PubMed]

5. Fiorina P, Shapiro AM, Ricordi C, Secchi A (2008) The clinical impact of islet transplantation. Am J Transplant 8: 1990-1997. [PubMed]

6. Ferber S, Halkin A, Cohen H, Ber I, Einav Y, et al. (2000) Pancreatic and duodenal homeobox 1 gene induces expression of insulin genes in liver and ameliorates streptozotocin-induced hyperglycemia. Nat Med 6: 568-572. [PubMed]

7. Takahashi K, Yamanaka S (2006) Induction of pluripotent stem cells from mouse embryonic and adult fibroblast cultures by defined factors. Cell 126: 663-676. [PubMed]

8. Zhou Q, Brown J, Kanarek A, Rajagopal J, Melton DA (2008) In vivo reprogramming of adult pancreatic exocrine cells to b-cells. Nature 455: 627 632. [PubMed]

9. Collombat $P, X u X$, Ravassard P, Sosa-Pineda B, Dussaud S, et al. (2009) The ectopic expression of Pax4 in the mouse pancreas converts progenitor cells into alpha and subsequently $\beta$ cells. Cell 138: 449-462. [PubMed]

10. Thorel F, Népote V, Avril I, Kohno K, Desgraz R, et al. (2010) Conversion of adult pancreatic alpha-cells to beta-cells after extreme beta-cell loss. Nature 464: 1149-1154. [PubMed]

11. Xu X, D'Hoker J, Stangé G, Bonné S, De Leu N, et al. (2008) B cells can be generated from endogenous progenitors in injured adult mouse pancreas. Cell 132: 197-207. [PubMed]

12. Konno M, Hamabe A, Hasegawa S, Ogawa H, Fukusumi T, et al. (2013) Adipose-derived mesenchymal stem cells and regenerative medicine. Dev Growth Differ 55: 309-318. [PubMed]

13. Arda HE, Benitez CM, Kim SK (2013) Gene regulatory networks governing pancreas development. Dev Cell 25: 5-13. [PubMed]

14. Jonsson J, Carlsson L, Edlund T, Edlund H (1994) Insulin-promoter-factor 1 is required for pancreas development in mice. Nature 371: 606-609. [PubMed]

15. Kajiyama H, Hamazaki TS, Tokuhara M, Masui S, Okabayashi K, et al. (2010) Pdx1-transfected adipose tissue-derived stem cells differentiate into insulinproducing cells in vivo and reduce hyperglycemia in diabetic mice. Int $\mathrm{J}$ Dev Biol 54: 699-705. [PubMed]

16. Xu H, Tsang KS, Chan JC, Yuan P, Fan R, et al. (2013) The combined expression of Pdx1 and MafA with either Ngn3 or NeuroD improves the differentiation efficiency of mouse embryonic stem cells into insulin-producing cells. Cell Transplant 22: 147-158. [PubMed]
17. Naya FJ, Stellrecht CMM, Tsai MJ (1995) Tissue-specific regulation of the insulin gene by a novel basic helix-loop-helix transcription factor. Genes Dev 9: 1009-1019. [PubMed]

18. Kim JW, Seghers V, Cho JH, Kang Y, Kim S, et al. (2002) Transactivation of the mouse sulfonylurea receptor I gene by B2/NeuroD. Mol Endocrinol 16 1097-1107. [PubMed]

19. Yeung TY, Seeberger KL, Kin T, Adesida A, Jomha N, et al. (2012) Human mesenchymal stem cells protect human islets from pro-inflammatory cytokines. PLoS One 7: e38189. [PubMed]

20. Tan J, Wu W, Xu X, Liao L, Zheng F, et al. (2012) Induction therapy with autologous mesenchymal stem cells in living-related kidney transplants: a randomized controlled trial. JAMA 307: 1169-1177. [PubMed]

21. Blanc KL, Mougiakakos D (2012) Multipotent mesenchymal stromal cells and the innate immune system. Nat Rev Immunol 12: 383-396. [PubMed]

22. Peng Y, Ke M, Xu L, Liu L, Chen X, et al. (2013) Donor-derived mesenchyma stem cells combined with low-dose tacrolimus prevent acute rejection after renal transplantation: a clinical pilot study. Transplantation 95: 161-168. [PubMed]

23. Reinders ME, de Fijter JW, Roelofs H, Bajema IM, de Vries DK, et al. (2013) Autologous bone marrow-derived mesenchymal stromal cells for the treatment of allograft rejection after renal transplantation: results of a phase I study. Stem Cells Transl Med 2: 107-111. [PubMed]

24. González MA, Gonzalez-Rey E, Rico L, Büscher D, Delgado M (2009) Adiposederived mesenchymal stem cells alleviate experimental colitis by inhibiting inflammatory and autoimmune responses. Gastroenterology 136: 978-989. [PubMed]

25. Du Z, Wei C, Cheng K, Han B, Yan J, et al. (2013) Mesenchymal stem cellconditioned medium reduces liver injury and enhances regeneration in reduced-size rat liver transplantation. J Surg Res 183: 907-915. [PubMed]

26. Ohmura Y, Tanemura M, Kawaguchi N, Machida T, Tanida T, et al. (2010) Combined transplantation of pancreatic islets and adipose tissue-derived stem cells enhances the survival and insulin function of islet grafts in diabetic mice. Transplantation 90: 1366-1373. [PubMed]

27. Hansson M, Tonning A, Frandsen U (2004) Artifactual insulin release from differentiated embryonic stem cells. Diabetes 53: 2603-2609. [PubMed]

28. Kawamoto K, Konno M, Nagano H, Nishikawa S, Tomimaru Y, et al. (2013) CD90- (Thy-1-) high selection enhances reprogramming capacity of murine adipose-derived mesenchymal stem cells. Dis Markers 35: 573-579. [PubMed]

29. Rovira M, Scott SG, Liss AS, Jensen J, Thayer SP, et al. (2010) Isolation and characterization of centroacinar/terminal ductal progenitor cells in adult mouse pancreas. PNAS 107: 75-80. [PubMed]

30. Okita K, Nakagawa M, Hyenjong H, Ichisaka T, Yamanaka S (2008) Generation of mouse induced pluripotent stem cells without viral vectors. Science 322 : 949-953. [PubMed]

31. Miyoshi N, Ishii H, Nagano H, Haraguchi N, Dewi DL, et al. (2011) Reprogramming of mouse and human cells to pluripotency using mature microRNAs. Cell Stem Cell 8: 633-638. [PubMed]

32. Anokye-Danso F, Trivedi CM, Juhr D, Gupta M, Cui Z, et al. (2011) Highly efficient miRNA-mediated reprogramming of mouse and human somatic cells to pluripotency. Cell Stem Cell 8: 376-388. [PubMed]

33. Noguchi H, Bonner-Weir S, Wei FY, Matsushita M, Matsumoto S (2005) BETA2/NeuroD protein can be transduced into cells due to an arginine- and lysine-rich sequence. Diabetes 54: 2859-2866. [PubMed]

34. Sakano D, Shiraki N, Kikawa K, Yamazoe T, Kataoka M, et al. (2014) VMAT2 identified as a regulator of late-stage $\beta$-cell differentiation. Nat Chem Biol 10 : 141-148. [PubMed] 